\title{
Authoring tools for network-based training
}

\author{
E. McDaid, G. Row, W. O'Brien and A. Hashim \\ School of Computing, Information Systems and Mathematics, \\ South Bank University \\ 103 Borough Road, London, SE1 OAA, UK \\ E-mail: mcdaideg@sbu.ac.uk
}

\author{
M. McCool \\ INCORE \\ University of Ulster, Magee College, Londonderry, BT48 7JL, \\ $U K$
}

\begin{abstract}
This paper presents an overview of the TALENT toolkit--a software architecture and set of software tools designed for producing and delivering multimedia training material over computer networks. The toolkit addresses many current problems relating to multimedia development and delivery including conversion of existing materials, reusability, internationalisation, customisation, intellectual property right tracking, support for multiple developers and abstraction neutrality. Organisational and commercial requirements are also addressed. The toolkit has been designed from the outset to be network-centric and the development tools themselves can operate over a network. Issues relating to the design and implementation of the toolkit are discussed. A simple model of multimedia systems is presented and the main components of the toolkit are described. The toolkit would appear to represent a significant advance in our ability to develop and deploy multimedia training over networks.
\end{abstract}

\section{Keywords}

Training, WWW, Courseware, Java, Authoring, Interactive multimedia 


\section{INTRODUCTION}

Recent advances in computer technology such as improvements in the multimedia capabilities of personal computers, the World-Wide Web and its wide range of associated technologies together with faster computer networks have made network-based training a technologically viable option. These factors, in conjunction with the increasing adoption of the Internet and corporate intranets by commercial organisations, have led to significant interest in the use of networkbased training as a cost-effective mechanism for training delivery.

Given the size of the training industry, let alone education in general, and the huge investment in existing training materials these developments represent a significant opportunity as well as a threat to those organisations currently engaged in training. The opportunity lies in the possibility of exploiting wider markets and new business areas while the threat lies in any failure to make effective use of these opportunities.

The TALENT project (Training and Learning Environment with Networked Tutoring) which is funded under the ESPRIT IV programme of the European Commission has the goal of providing a complete sofiware environment for training development and delivery as well as network-tutoring in order to support a distributed virtual training centre. In addition, the environment will support the supply chain issues of the network-based training industry of the future.

The TALENT consortium is composed of representatives of each part of the training supply chain from courseware developers through training providers, localisers, resellers and consumers as well as training technology developers.

This paper is concerned with Phase I of the TALENT project which is nearing completion. This has included the development and use of a prototype software toolkit for producing and delivering multimedia network-based training. We will describe the design and development of the toolkit together with it's associated software architecture but first we will discuss some of the issues which the toolkit addresses.

\section{INTERACTIVE MULTIMEDIA}

Many of the potential benefits claimed for network-based training have been cited in the past for more traditional forms of interactive multimedia training such as that delivered using Interactive videodisc, CD-I and CD-ROM technologies (Fletcher, 1996). It is argued that interactive multimedia enhances the learning process through engaging the student while the use of multiple simultaneous media improves information transfer and retention. In addition, there are potential economic benefits for the organisation through reduction in the requirement to travel and lower costs associated with self-paced learning. While there is evidence 
to support these claims (Fletcher, 1996), the widespread adoption of interactive multimedia in training which was predicted has failed to materialise for a number of reasons.

A major hurdle to the adoption of multimedia training has been the high costs associated with the production of multimedia titles. Estimates vary but guidelines for multimedia development indicate that it can require between 50 to 600 hours of development time to produce one hour of multimedia training (Golas, 1993). This is in contrast to conventional tutor-led training which may require 6 to 20 hours of preparation time for a one hour training session. Other factors include multimedia development skills which are in short supply and demand a premium. The expense of acquiring and supporting specialist multimedia development software and hardware is another significant obstacle. For these reasons, embarking on a first multimedia development project can present a daunting prospect to many organisations particularly from the perspective of cost.

Existing authoring systems used for multimedia development can require considerable expertise in order to be used effectively. This is due to the fact that they are, in the main, general purpose multimedia development tools rather than being geared specifically to a particular problem. As such, authoring systems contain many features both in their scripting languages as well as in their developer environment which are seldom or never used by the majority of developers. These features do, however, add significantly to the learning-curve for the package as a whole. For example, the majority of authoring systems have internal tools for painting. These are seldom used by professional developers who prefer to use specialised painting tools even though they are external to the authoring environment.

The majority of existing authoring systems are intended for used by a single developer. This is a major drawback and in some ways quite surprising as multimedia development is often a team effort due to the size, complexity and required skills set of many multimedia projects. Single user development tools represent a significant bottleneck as they prevent the efficient use of workflow methodologies and may require additional integration phases in order that the efforts of individuals may be brought together to form a unified system. Such integration may be complicated in some authoring systems because of the paradigms which they employ. In any case, extensive use of stubs during development and glue during integration may be required.

Interchange of information or assets between different authoring systems is currently poorly supported. A variety of standards exist for individual media types such as text, sound, image and video data--indeed, several standards exist for most media types. A number of multimedia document standards have been proposed or are under development (Newcomb, Kipp and Newton, 1991, Kretz and Colaïtis, 1992), however, none are in widespread use at this time. As a result it can be difficult for information in one multimedia title to be reused in another unless the same authoring system is used. Even then this process may require significant integration effort as mentioned above. 
In addition to development skills it may also be necessary for the multimedia author to possess a certain degree of creative ability if titles are to embody professional design or exhibit high production values. By analogy, it is possible for anyone to buy a paintbrush but that does not mean that they will produce a masterpiece. Few individuals are talented in both technical and creative areas. As a result it is often necessary for multimedia productions to require teams of specialists such as programmers, designers and various creative types.

Cross-platform compatibility issues have been a major problem for multimedia development. While some tools provide support for multiple platforms it is rarely a straightforward process to develop cross-platform titles or to port from one platform to another after development. Problems often arise because the versions of the same authoring tool on different platforms are not completely compatible with each other. Also, there are often underlying technical problems such as the different ways in which colour is handled for example which can present traps for the inexperienced or unwary developer.

Another problem with traditional multimedia training material has been the requirement for specialised platforms for its delivery. Videodisc and CD-I machines have never been commonplace in the majority of commercial environments and even now multimedia PCs are not the normal specification for desktop workstations. In addition, some organisations discourage the purchase of computers with CD-ROM drives as these are often perceived as being used by employees solely for listening to music while they work.

Finally, interactive multimedia courseware despite its high development costs may become obsolete soon after production. This is due to the fact that optical disks which are used for distribution are not generally rewritable and there are significant overheads in the production of the various sorts of disks employed for delivery. Optical disks are still the only viable physical medium for the distribution of multimedia due to the large quantities of data involved. Mass production reduces the cost per unit but exacerbates the problem of changing a title to reflect changes in the course due to the additional mastering and pressing costs. This is, of course, on top of any additional authoring costs incurred.

Most of the problems we have just mentioned are applicable to all authoring systems. Many are to do with the way in which information is assembled and stored within the authoring environment or distributed to the consumer. Networkbased training may succeed in delivering on the promises of interactive multimedia training if it can overcome these and other problems.

\section{REQUIREMENTS}

The fundamental requirements of the TALENT toolkit were that it be capable of supporting the development and delivery of multimedia training over networks. From the above discussion it is clear that a number of other important requirements should be addressed if the toolkit was to be successful. Prime among these is the need to bring down the development costs associated with the production of 
multimedia courseware. Within the TALENT project we refer to this as 'moving from the Hollywood model to the newsroom model'. Costs can be reduced in a number of ways--many of which relate to the problems described above.

Network delivery of training in itself addresses issues of specialised platform requirements, cross-platform compatibility and obsolescence through physical distribution media. The increasing importance of networks is helping to break down compatibility barriers between different platforms through the use of standard network protocols--network delivery can therefore help to overcome cross-platform development problems. It also reduces the hardware requirements for the delivery platform. Indeed, low specification platforms such as the low-cost Network Computers which are currently under development are capable of use as network-based training delivery platforms. The problems of obsolescence associated with optical disks can be reduced through the use of hybrid technologies which enhance content on disks with live links to updated information on the network. There will probably be a requirement for disk based distribution for some time due to the limited bandwidths of many users network connections.

Ease of use is of crucial importance. The toolkit should be usable by individuals with little or no experience of multimedia development. This will ease the entry of organisations into multimedia development as they can use their existing staff without the need to reskill or replace them. Also, users will have less of a learning curve if usability is greater. The issues of design or creative ability can be addressed through the use of templates and through the inherent support provided by the network approach for a multi-user, multi-disciplinary team.

It is important to provide support for multiple developers and to improve the productivity of developers in general. Support for multiple developers could be achieved through using client-server technology for the toolkit itself. Improving productivity would be addressed through focusing the toolkit on the particular domain of multimedia training as opposed to trying to produce a general purpose authoring tool. Productivity could also be improved through better design of the tools such that they focus the efforts of the developer on the task of courseware development--particularly on the information necessary for the process.

Information interchange is a significant requirement for multimedia courseware. It is often necessary for assets or parts of courses to be reused in other courses either in an unmodified or modified form. It is also envisaged that there is the potential for increased traffic in assets and courseware between training organisations in the future training business marketplace. It is important to be able to track the use of assets for IPR purposes such as royalty payment and it may also be a requirement to support the watermarking of assets for the purpose of copyright identification.

In the absence of widespread standards for multimedia document interchange it was decided to take an open, abstraction neutral approach to information storage and organisation. This approach is equally applicable to card, script or time-line based paradigms. 
In addition to requirements indicated by existing authoring systems and their use a number of additional requirements were determined through surveys conducted by the consortium.

In order to protect investment in existing training materials a major requirement of the TALENT toolkit was that it provide support for the conversion of these materials most of which exist as paper-based or computer-based documents in a variety of formats. Obviously it is not possible to produce an interesting multimedia system from solely from a word-processor document. However, it is necessary to provide a means of reusing the text, image and other assets from which they are composed. In addition, much of this material is the result of extensive pedagogic design so it would also be useful for the resulting multimedia system to retain this instructional design in some way.

Support for internationalisation is increasingly important--particularly with the trend toward globalisation. Markets such as Europe and Asia are comprised of many linguistic, national and cultural groups so the ability to easily localise products such as training materials can have a significant impact on the size of their potential market. Therefore, it is important to provide facilities which support localisation such as the isolation of language sensitive information or the provision of support for different character sets.

In addition to the above, mostly technical, requirements a number of commercial requirements were identified including access control, logging and reporting, registration of students and enrollment for courses and so on. Such business services provide a realistic starting point for the integration of the toolkit in to a commercial training environment.

These requirements indicated that the TALENT toolkit would be significantly different from existing multimedia authoring systems as well as presentiñ an interesting set of technical challenges. In order to determine the best way to proceed we investigated the nature of multimedia systems and arrived at a simple yet useful model.

\section{A SIMPLE MODEL OF MULTIMEDIA}

Multimedia systems are fundamentally composed of atomic objects or assets which correspond to information in one of a number of formats such as text, audio, image, video and so on. While it may be possible to subdivide assets, for example, decompose a text string into characters or a video sequence into frames, it makes no sense as far as the holistic nature of the system is concerned to do so.

Assets are organised within a multimedia system in space and time such that, for example, a video may appear at a certain location on the screen at a particular time while being synchronised with an audio sound track that begins and ends at the same time as the video.

A number of abstractions are in common use which are intended to assist the developer as well as the user in the process of forming mental models of the multimedia system. These include timeline, card and script based abstractions as 
well as a number of others (Vaughan, 1996). Abstractions also serve to support the dialogue structure of the system in terms of differentiating and grouping screens, forming navigational structures and reinforcing spatial cues which assist in the formulation of cognitive maps.

However, ultimately all multimedia systems can be reduced to state based descriptions in which objects appear on the screen at a certain point in time, possibly move around, perhaps in response to user interaction and subsequently disappear from the screen at a later time. Accompanying phenomena such as audio can be treated in exactly the same way.

This abstraction neutral view of multimedia has the potential for supporting a certain level of multimedia document interchange between different authoring packages. As an illustration, it is possible to simulate a card-based metaphor in a timeline-based authoring system and vise versa. Abstraction neutrality was a central concept in the ScriptX system (Pinheiro, 1994). However, the advent of Java led to the demise of the ScriptX project.

Such a reductionist view leads us to a simple model of multimedia sysiems which focuses on the information which they contain. We see multimedia systems as being composed of three elements:

1.Content;

2.Structure;

3.Presentation.

'T he first point is simple--multimedia systems contain a set of assets from which they are composed. The structure is the way in which these assets are organised within the system and this can be made to reflect any abstraction which can be imagined. Structure is different from presentation in the sense that the structure specifies which objects occur on a particular screen but the presentation may describe where they are, what size and what color.

Using this model it is possible for us to develop systems in which content, structure and presentation may be treated separately. This has a number of important advantages. For example, it is possible to apply different structures to the same content in order to impose different pedagogic principles on a particular course. It is also possible to apply different presentation templates to a given set of structure and content for the purpose of tailoring a title to a particular organisation or audience. A major advantage lies simply in the ability to work on each element in isolation while the approach itself greatly encourages the reuse of assets and structure components.

This simple model which is similar in some respects to that used in the Open Document Architecture standard (Appelt, 1991) greatly simplifies the authoring process and has the potential to greatly reduce the effort and costs associated with multimedia courseware development. 


\section{CONTENT}

The content of a multimedia system is the set of assets from which it is composed. As we have seen assets can be pieces of text, images, sounds, video, music and so on. The TALENT toolkit must allow developers to store, browse, search for and update assets in any of these forms. This requires the storage of assets as well as information relating to these assets such as name, owner, description, creation date and so on.

As the system may contain a large number of assets it is important to provide mechanisms for finding and organising assets according to user defined criteria.

In order to encourage reuse and to support multiple developers it is important that all assets are available to each developer. However, the concept of ownership is important so that one developer does not remove or modify assets that belong to someone else. This protects the integrity of each developers work.

\section{STRUCTURE}

The structure of a multimedia document describes how the assets are combined and presented to the user. It is the structure which specifies both the makeup of the individual screens, windows, cards or whatever and the navigational paths which allow the user to move between them.

The TALENT toolkit takes an abstraction neutral approach to structure by allowing for the creation and storage of arbitrarily complex structures within the system. These structures can be created and manipulated through the use of tools. It is possible to provide a range of tools each of which is optimised for a specific kind of structure -for example, trees graphs, tables, lists and so on. Because a single internal representation is used it is possible to add new tools in order to support new kinds of structures and thus to extend the toolkit without introducing incompatibilities.

Three basic kinds of structures are central to the current implementation of the toolkit. These are: a tree based hierarchy which reflects the overall organisational structure of a piece of courseware; a graph based navigational structure which is driven by pre-requisites in order to implement a pedagogic model; and a list based content structure which maps assets onto particular screens. This last structure is also used to map assets onto templates which, as we shall now see, can change the way in which they are presented.

\section{PRESENTATION}

Templates provide a useful means of changing the presentation of information while keeping the content the same. Presentation graphics packages such as Powerpoint use templates to provide different layouts, backgrounds, colour 
schemes and typography while the information in the presentation remains the same.

TALENT templates are more complex as the number, order and format of the assets which go to make up screens can vary. For this reason, TALENT templates are really families of templates with each asset in a particular screen having a template type. The template type then maps on to a range of particular template instances corresponding to media formats for which that template type is valid. In addition there are template types for complete screens as well as for structures of assets such as lists and tables. At this time templates are not created by the user but a range of templates are provided.

TALENT templates are complex but the underlying principle is simple. The developer can change how an asset is rendered by specifying a given template for that asset. A complete description of TALENT templates is beyond the scope of this paper.

\section{ARCHITECTURE}

The TALENT toolkit takes an information-centred view of multimedia and the software architecture which was developed to support the toolkit reflects this. Central to the architecture is a database in which all assets, as well as structures, templates and other information are stored. The software tools act as an interface between the developer and the database for the purpose of inserting, modifying and accessing the various kinds of information. Figure 1 shows an overview of the TALENT architecture.

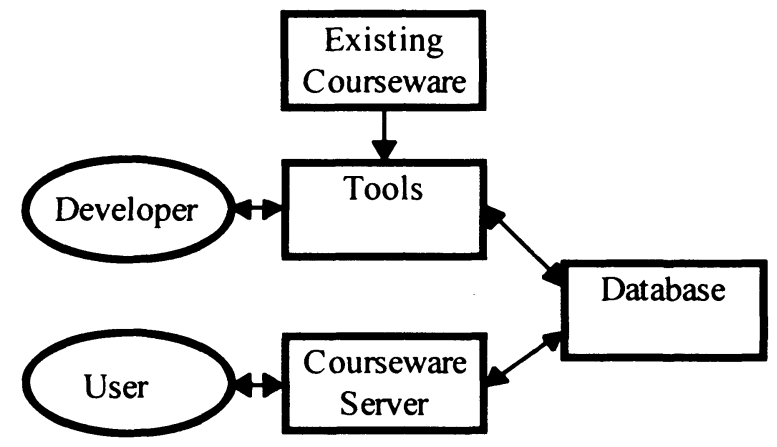

Figure 1. Overview of TALENT Architecture.

The World Wide Web is used as a development environment and delivery vehicle for the toolkit as well as the courseware which is produced using it. This 
affords considerable flexibility as both developers and students can access the courseware via a network.

The majority of the toolkit has been developed using Java and it is currently implemented as a set of Java Applets. This goes a long way towards making the toolkit cross-platform as it can be accessed from any machine that supports a Java enabled WWW browser.

The database is a modified version of Msql which is a light weight database server implementing a useful subset of ANSI SQL. This has been extended to cope with binary large object (BLOB) data in order to be able to store assets such as images and sounds. It should be an easy matter to modify the toolkit in order for it to work with commercially available database products such as those produced by companies like Oracle.

The database is capable of storing many courses or versions of courses simultaneously. As these courses may share assets and structures it encourages reuse in an efficient manner. It also makes it easy to localise a course simply by providing replacements for language sensitive assets.

The courseware server is currently a CGI extension to a WWW server and courseware appears to the users as a series of WWW pages. The WWW pages are created dynamically using assets, structure and templates extracted from the database.

In addition to courseware, the database is also used to store a wide range of administrative and housekeeping information includi, student records and registration.

The database is also used to implement a virtual file system which allows multiple developers to store and organise information in ways that can be shared and which encourage the reuse of assets and sections of courses.

The TALENT toolkit consists of a number of major components:

1.Courseware converter;

2.Asset manager;

3.Filing system;

4.Structure editors;

5.Template editor;

6.Integrity checker;

7.Courseware server.

The courseware conversion tools are described elsewhere (McDaid et al, 1997). Additional tools provide support for administrative and tutoring functions but these will not be discussed here. We will now look at a few of these tools in more detail.

\section{TOOLS}

Figure 2 shows the asset tool which is central to the management and organisation of content. This is divided into two parts: the left side relating to assets and the right side interfacing to the virtual file system. 
The list on the left presents a subset of all of the assets in the database. The contents of this list are modified using the asset filter dialogue (see below). An asset in this list may be selected for viewing, displaying information or editing as appropriate. New assets may be uploaded into the database using a separate dialogue. This allows the developer to select a file on their desktop computer which is then uploaded through the HTTP protocol. A set of format conversion filters can be applied to convert the format of a particular asset to a standard format at this stage. The current filter set supports many popular image and sound formats.

The virtual file system implements a hierarchy of folders within the database which provide user space for each developer. The option button (top right) displays the path within the file system and is one of the ways in which the user can change directory. The list below shows the assets and folders within a given folder. Assets are not physically copied into folders but rather references to them so the folder system imposes a low overhead on the database. Also it is not possible to delete an asset simply by removing all of its folder entries. The copy button allows assets to be copied into folders while the buttons at the bottom allow folders to be created, deleted and permits the user to change directory. A separate folder tool (not shown) permits users to copy and move folders and assets around the file system.

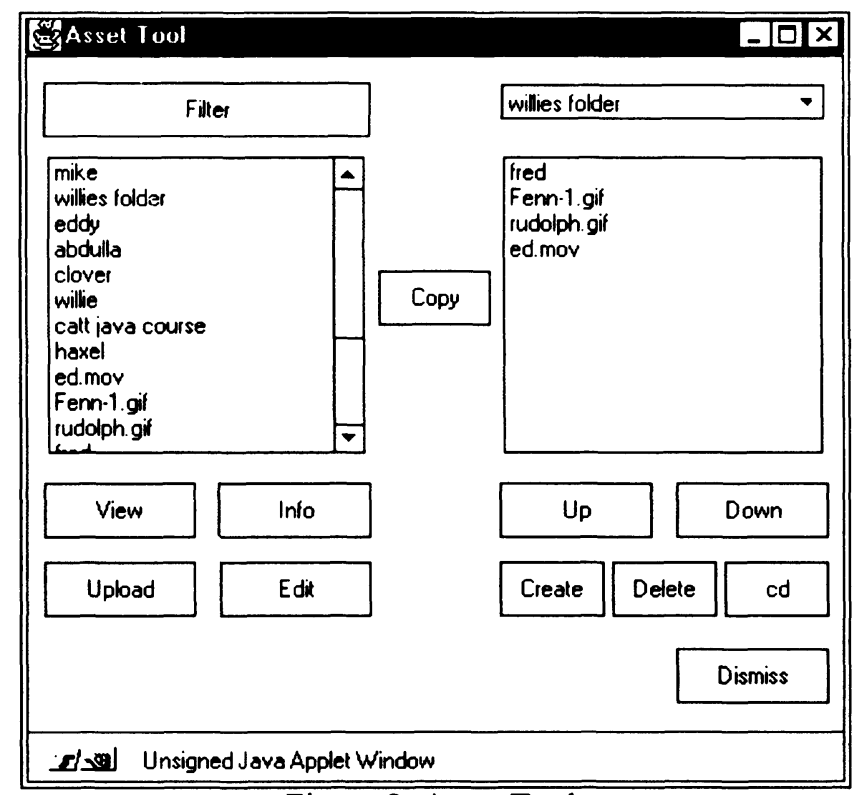

Figure 2. Asset Tool.

The asset filter is shown in figure 3. This allows the developer to select a subset of assets from the database for use within the asset tool. This dialogue allows the 
user to construct a query filter based on a variety of criteria. The results of this query are displayed in the left hand field of the asset tool.

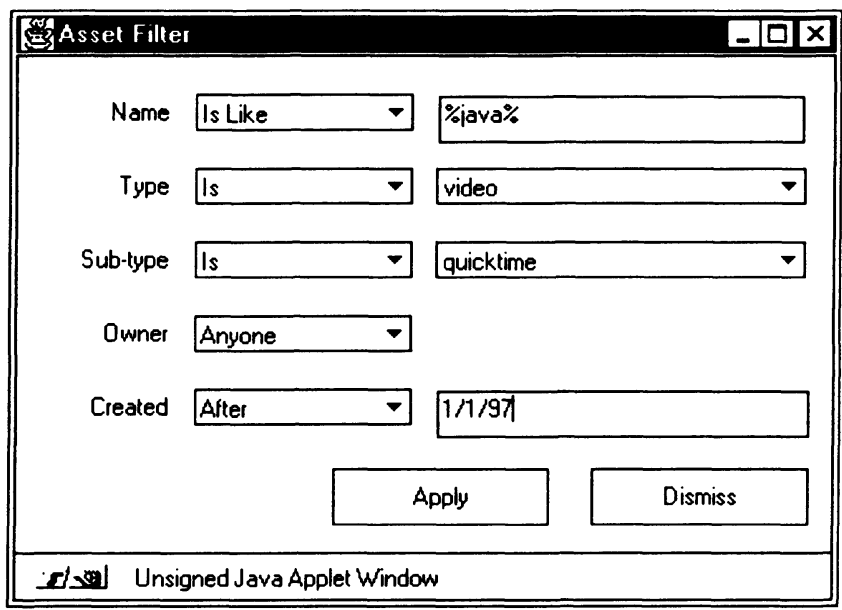

Figure 3. Asset Filter.

A number of tools allow the developer to create and manipulate the various structures within the database. Figure 4 shows the structure editor which displays the overall structure of a piece of courseware as a tree.

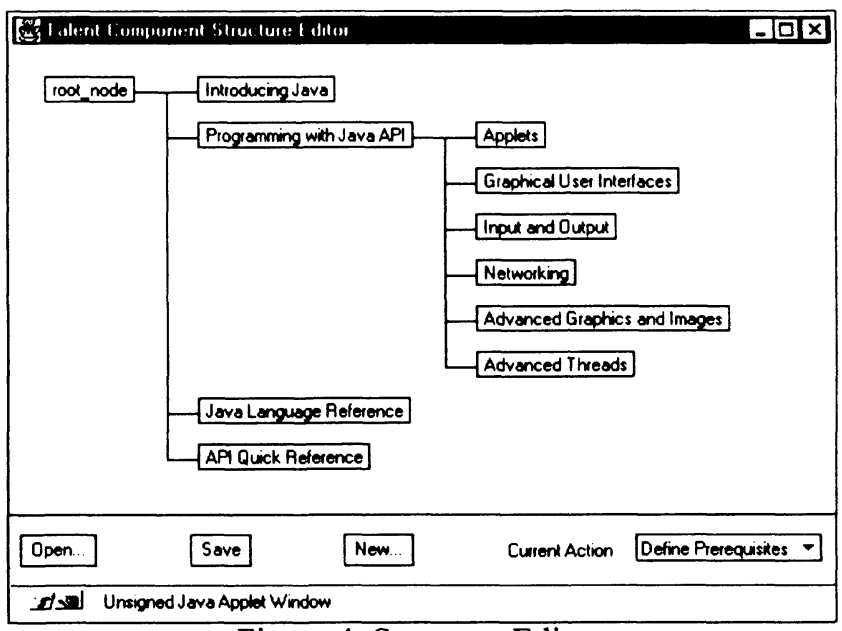

Figure 4. Structure Editor.

This has all of the usual features associated with tree editors including the ability to move nodes around within the hierarchy as well as the ability to fold and expand branches. Nodes can be added, deleted or modified. 
Hierarchies can be created from new or are produced when an existing piece of courseware is converted. It is possible to copy some or all of a hierarchy for inclusion into a different course. Such a copy may simply be a reference in which case it cannot be changed or the hierarchy may be cloned and the cloned copy can then be edited. Audit trails record the provenance of such structures to prevent undesirable conditions such as referenced hierarchies being edited or deleted. Similar measures are taken to prevent assets being deleted if they are being used in any course.

The hierarchy diagram presents one aspect of the navigational structure of a course. Fine control over the pedagogic structure is expressed through the graph tool (Figure 5).

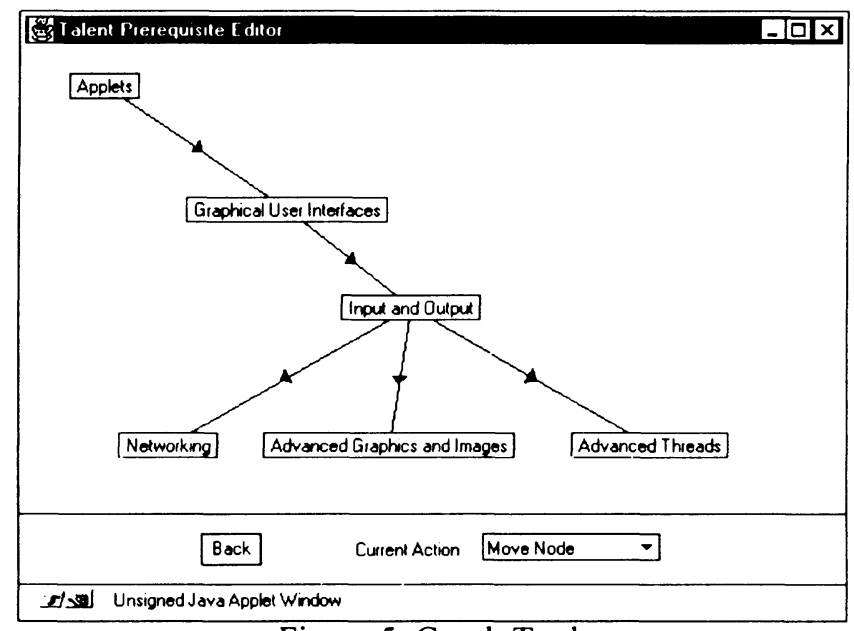

Figure 5. Graph Tool.

The graph tool allows the developer to describe the pre-requisites which apply within a course by specifying which nodes must be completed before a particular node may be attempted. The concept is similar to that of a PERT diagram as used in project management tools.

The nodes within the graph tool are the same nodes that occur in the hierarchy tool and together they allow the designer to specify the possible navigational structures in abstract terms. Thus, it is not necessary for each possible path through the system to be explicitly defined as any path which meets the criteria is possible. This allows the student to access the courseware in a flexible way which can be tailored by the student or developer to meet particular interests or needs. The dialogue structures are enforced by the courseware server which provides the necessary additional navigational controls to permit the student to navigate the course. 


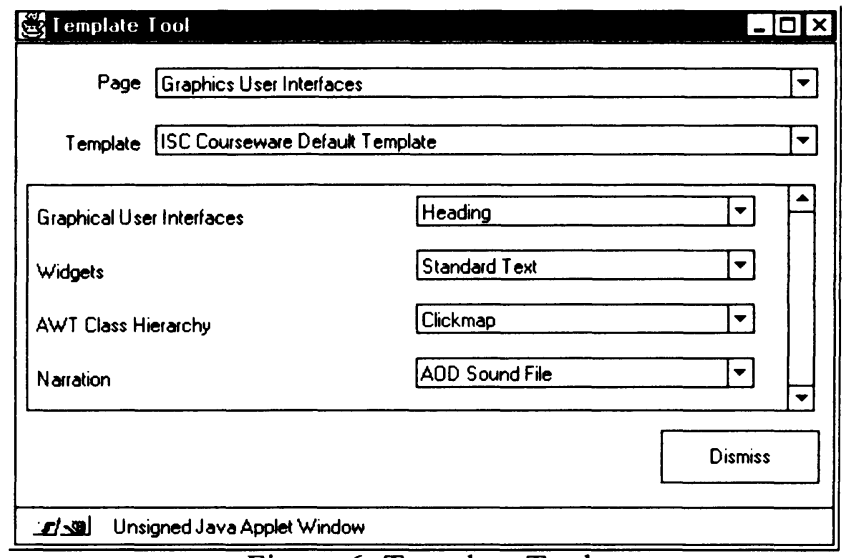

Figure 6. Template Tool.

The template tool (Figure 6) permits the designer to change the way in which the assets which go to make up a particular page are presented. The page template specifies a set of templates which can be applied to each of the asset types present. It also controls reordering and layout of components on a rage as well as specifying global variables which in turn effect the individual asset templates. Each asset type has an associated set of templates including a default template which is used if no template type is specified. In simple terms templates can be thought of as HTML wrappers which change the way in which assets are rendered onto WWW pages. However, the implementation is more complicated.

This brief overview is intended to provide a flavour rather than an in depth description of the tools. The toolkit contains a large number of other tools and interested readers may wish to refer to the TALENT toolkit user guide for a more detailed description of the software (McDaid, 1997).

\section{DISCUSSION}

The TALENT toolkit has been implemented and used within the consortium to produce a number of courseware modules. Some of these have been converted from existing courses while others have been produced from new. It has also been used to localise some of these courses into different European languages.

It is apparent that the implementation is still a prototype and considerable refinement would be required before this software could be turned into a commercial product. However, the approach would appear to be promising. In particular, the use of the Web as an authoring environment has significant benefits including cross-platform delivery, support for multiple developers, reusability and internationalisation. The information-centric model is interesting in its ability to overcome the current lack of multimedia document standards. Compatibility with 
emerging standards can be easily provided and a certain degree of interoperability with conventional authoring systems is also possible.

The TOOLKIT will continue to develop throughout the project in response to feedback from users as well as advances in its underlying technologies. The next phase of the TALENT project will see the addition of tutoring facilities based on text-based chat and video-conferencing. It is likely at this stage that the delivery mechanism will evolve beyond HTML to a more advanced and interactive software layer but it is too early to predict how the technologies that this will be based on will develop. Later development of the toolkit will focus on the supply chain and organisational functions supported by the tools. However, it is envisaged that technological enhancement will continue throughout the project.

\section{CONCLUSIONS}

The TALENT toolkit addresses a number of important problems in the development of network-based multimedia courseware. By adopting a networkbased architecture it provides improved support for cross-platform development and for multiple developers. The model overcomes a number of problems in conventional authoring systems particularly in the interchange, reuse and internationalisation of courses and assets. The toolkit is simple and easily extended and as such could provide the basis for important future work in this field.

\section{REFERENCES}

Appelt, W. (1991) Document Architecture in Open Systems, The ODA Standard. Springer-Verlag, New York.

Fletcher, J.D. (1996) Does This Stuff Work? Some Findings from Applications of Technology to Education and Training. Proceedings of Conference on Teacher Education and the Use of Technology Based Learning Systems. Warrenton, VA: Society for Applied Learning Technology.

Golas, K.C. (1993) Estimating Time To Develop Interactive Courseware In The 1990s, Proceedings of the 15th Interservice/Industry Training Systems and Education Conference, Orlando, Florida, November 29-December 2, 1993.

Kretz, F. and Colaiitis, F. (1992) Standardizing Hypermedia Information Objects, IEEE Communications Magazine, p60, May 1992.

McDaid, E., Row, G., McCool, M, Hashim, A. and O'Brien, W. (1997) Converting existing training materials to multimedia. Proceedings of Multimedia and Hypermedia Systems 97, Opatija, Croatia, 19-23 May, 1997.

McDaid, E. (1997) The TALENT Toolkit User Guide. TALENT Consortium Document 05R0010A, Interactive Systems Centre, South Bank University.

Newcomb, S., Kipp, N. and Newton V. (1991) The HyTime Hypermedia/Timebased Document Structuring Language, Communications of the ACM, p67, November 1991. 
Pinheiro, E.J. (1994) New Tools for Multimedia Development: ScriptX. 1994 CAUSE Annual Conference. Orlando, Florida, November 29-December 2, 1994.

Vaughan, T. (1996) Multimedia: Making It Work, 3nd Ed., McGraw-Hill, New York.

\section{BIOGRAPHY}

Eddy McDaid is a computer scientist who has been involved in human-computer interaction research since 1983. During that time he has worked at various centres of excellence in the UK on research and consultancy projects addressing $\mathrm{HCI}$, KBS, multimedia and distributed systems. Current interests include Java, visual programming, multimedia authoring tools and Web exploration technology. 